\title{
Evolution of ophthalmic and electrophysiological findings in identical twin sisters with the carbohydrate deficient glycoprotein syndrome type 1 over a period of 14 years
}

\author{
I Casteels, W Spileers, A Leys, L Lagae, J Jaeken
}

\begin{abstract}
Aims- To evaluate the evolution of ocular and electroretinographic findings in identical twin sisters with the carbohydrate deficient glycoprotein (CDG) syndrome over a period of 14 years.

Methods- Both girls underwent a clinical ophthalmic examination with funduscopy and an electrophysiological assessment with recording of flash electroretinogram (FERG) at the age of 4 years and 18 years Results-On ophthalmic examination at the age of 4 years an alternating convergent squint and a saccadic pursuit was diagnosed. In both, vision was $6 / 9$ bilaterally. Fundus examination showed normal optic discs, narrow blood vessels, and a mild irregular pigmentation in the periphery. In one girl the FERG showed a recognisable $a, b 1$, and b2-wave with reduced amplitude to less than $40 \%$ of the normal. In the other girl the reduction in amplitude was still more obvious, but for the white flash a small b1-wave was still present. At the age of 18 vision had remained $6 / 9$ in both eyes. Funduscopy showed pink optic discs, moderately narrowed blood vessels, and bony spicule pigmentary deposits in the mid periphery. The adapto ERG, performed in identical conditions at 18 years of age, showed a completely extinguished trace for both eyes.
\end{abstract}

Conclusions-Despite progressive deterioration of ERG findings good central vision was preserved over 14 years.

(Br F Ophthalmol 1996;80:900-902)

University Hospitals of Leuven, Belgium

Department of Ophthalmology I Casteels W Spileers A Leys

Department of Paediatrics

L Lagae

J Jaeken

Correspondence to: Ingele Casteels, Department of Ophthalmology, St Rafael UZ Leuven, Capucijnenvoer 33, B-3000 Leuven, Belgium

Accepted for publication 24 May 1996 retinal degeneration, and electrophysiological abnormalities have been described in patients with the CDG syndrome type $1 .^{3-6}$ We report the evolution of the ocular and electroretinographic findings in twin girls (PA and PS) with this CDG syndrome over a period of 14 years.
Patients, methods, and results

Twin girls aged 19 months presented to the department of paediatrics with psychomotor retardation and internal strabismus. Increased protein was found in the cerebrospinal fluid. The association of abnormalities in a number of glycoproteins and the subsequent finding of pathological transferrin heterogeneity led to the hypothesis of a defect in their common carbohydrate moiety. ${ }^{78}$ This was confirmed by the demonstration of a partial deficiency of sialic acid, as well as galactose and $N$-acetylglucosamine in serum transferrin and total serum glucoproteins. ${ }^{9}$

Both girls presented to the ophthalmic department at the age of 4 years. They had an alternating convergent squint; eye movement examination showed saccadic pursuit. There was no refractive error. Vision was $6 / 9$ in both eyes. Fundus examination showed normal optic discs and narrow blood vessels, and in the periphery a mild irregular pigmentation could be seen. Full field flash electroretinograms (FERG) were obtained after pupil dilatation with $15 \%$ phenylephrine and $1 \%$ cyclopentolate hydrochloride. For recording, bipolar contact lens electrodes were used with a ground electrode on the forehead. At that time only an adapto ERG was performed: after initial bleaching the ERG response to a white flash (white in Fig 1A) and immediately thereafter to a dim orange flash (using a Wratten orange number 26 filter) was recorded ( $0^{\prime}$ in Fig 1A). With this method isolation of the cone response is possible. In normal subjects a response with an a-wave and a b1-wave is present. After $15^{\prime}$ of dark adaptation, the response to the orange flash was recorded $\left(15^{\prime}\right.$ in Fig 1A): in normal eyes an a-wave, b1 cone wave, and a later b2 rod wave can be demonstrated clearly. ${ }^{10}$ For subject PA (Fig 1A) the responses were present with a recognisable $a, b 1$, and $b 2$-wave. The amplitudes were, however, clearly reduced to less than $40 \%$ of the normal. For subject PS (Fig 1A) the reduction in amplitude was more obvious: for the white flash a small b1-wave was still present; using the orange flash, no clear response was present. At the age of 7 both sisters had a vision of $6 / 9$ in both eyes.

Eye motility, squint, and funduscopy were unchanged. There was no refractive error. The girls were reassessed at the age of 18 . They were mentally retarded and wheelchair bound. 

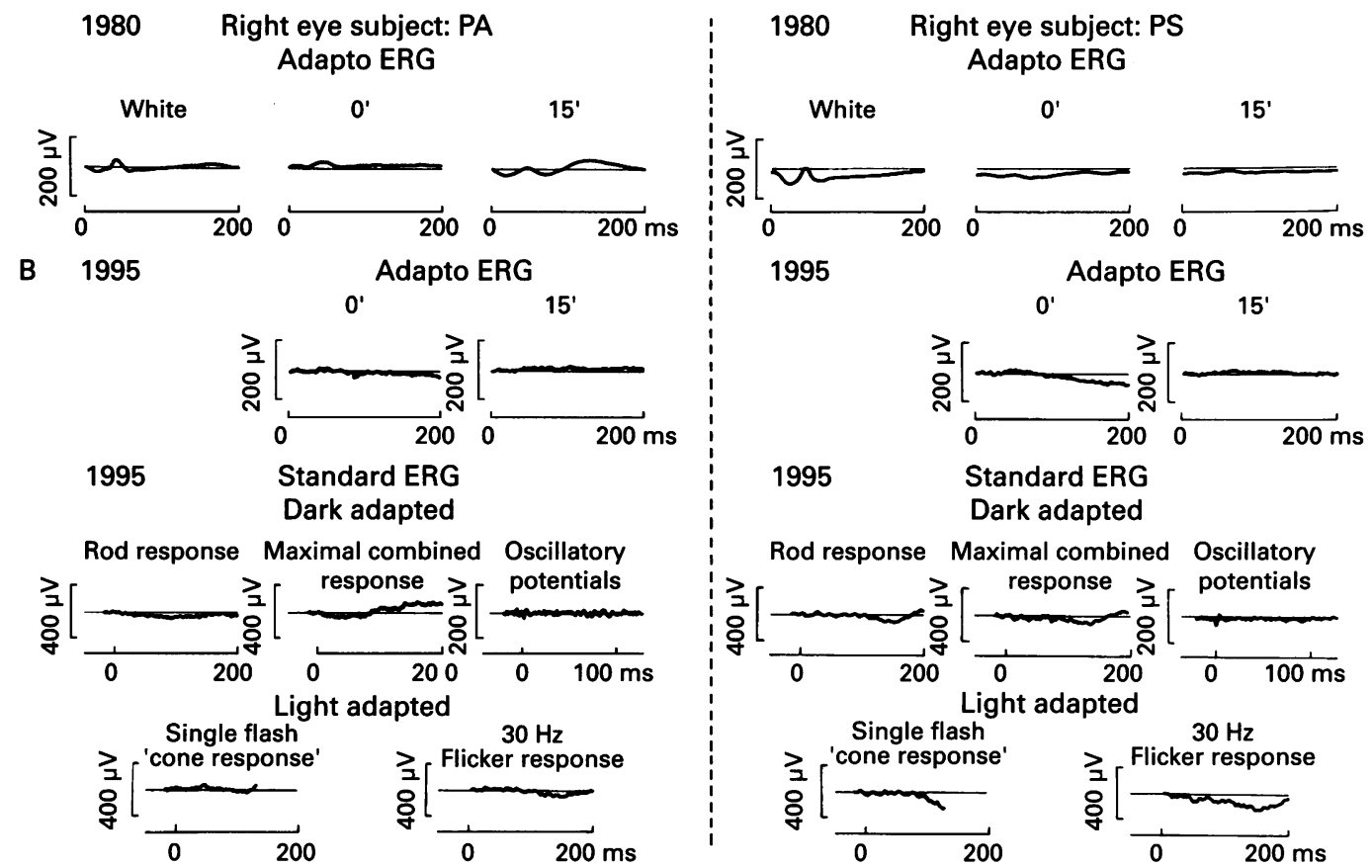

Figure 1 Evolution of electroretinographic findings in identical twin sisters (PA and PS) with the carbohydrate deficient glycoprotein syndrome type 1 at 4 years of age $(A)$ and 18 years of age $(B)$. (A) 1980: Response with reduced amplitude for both patients. (B) 1995: Adapto ERG, performed in identical conditions to 14 years ago, showed a completely extinguished trace for both patients. The ERG was now complemented with an ERG following the ISCEV standard ${ }^{11}$ : no recordable response was present.

Their parents did not note severe loss of vision or night blindness. On examination, vision had remained $6 / 9$ bilaterally for distance and near. Saccadic pursuit was still obvious. Ishihara colour vision testing was normal. Peripheral visual fields were found to be constricted on confrontation test. On funduscopy the optic discs were pink, blood vessels were moderately narrowed, and bony spicule pigmentary deposits could be seen in the mid periphery, being more evident in PS. Wrinkling of the macular retinal surface was seen in PA (Fig 2).

Now the adapto ERG, performed in identical conditions at 18 years of age showed a completely extinguished trace for both eyes. The ERG examination was now complemented with an ERG following the ISCEV standard $^{11}$ (Fig 1B): no recordable dark adapted or light adapted response was present. ${ }^{11}$

\section{Discussion}

Ocular findings in the carbohydrate deficient glycoprotein (CDG) syndrome type 1 have been reported as squint, retinal degeneration, electroretinographic, and visual evoked potential abnormalities. ${ }^{3-6}$ Very recently the importance of CDG syndromes as metabolic causes of retinal dystrophy with bone spicule pigment was stressed. ${ }^{6}$ Electrophysiological findings in patients with CDG syndrome have been described extensively by Andreasson et $a l .{ }^{5}$ Recordings were suggestive of a progressive tapetoretinal disorder with defined alterations in the ERG in every patient studied with this syndrome.

Our patients presented with an alternating convergent squint at the age of 19 months.
They had bilateral deficient abduction with a jerk nystagmus on attempted lateral gaze. There was no smooth pursuit, but saccadic eye
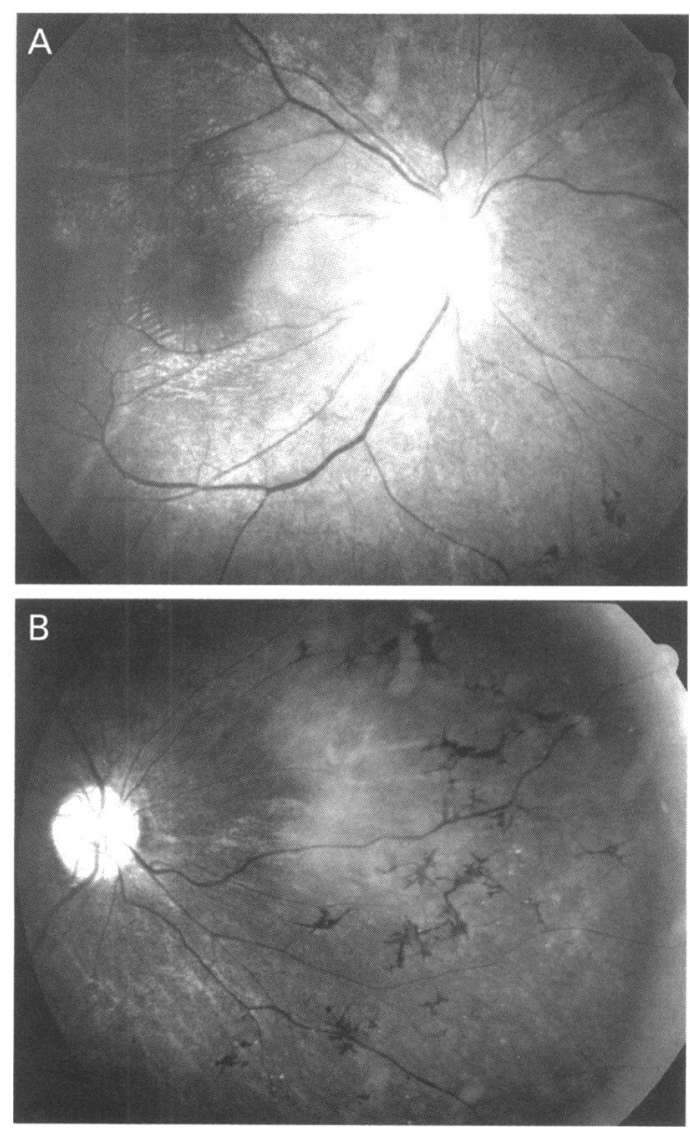

Figure 2 Fundus appearance in PA (A) and PS (B) at 18 years of age. Pink optic discs with narrowed blood vessels and bony spicule pigmentary deposits in the mid periphery. Macular retinal surface wrinkling in $P A(A)$. 
movements developed when following an object. All 29 patients described in a clinical overview by Jaeken $e t$ al had the same ophthalmic presentation in their first year of life. ${ }^{2}$

Funduscopic changes in the two girls at the age of 4 were typical for retinitis pigmentosa with narrowed vessels and mild pigment dispersion. The electroretinographic findings were strongly suggestive for retinitis pigmentosa. At that age central vision was $6 / 9$ in both girls. Fourteen years later, at the age of 18 , central vision had remained the same. Visual field examination with confrontation test showed constricted peripheral fields. On funduscopy pigmentary changes had clearly been progressive over 14 years with the development of spiculae in the mid periphery. The ERG also showed progression of retinal dystrophy with absence of rod and cone function. Retinitis pigmentosa in our patients was clearly progressive as shown by funduscopic pigmentary changes and by electroretinographic findings. The ERG responses decreased markedly over 14 years.

Andreasson et al described the electrophysiological findings in a 48-year-old patient with 'useful' central vision. ${ }^{5}$ In this patient the ERG showed some residual cone function. They stated that progressive disease does not necessarily result in blindness. Central vision remained unchanged in our patients over 14 years, despite obvious deterioration of the FERG response. This is the first report of repeated electroretinographic findings and vision measurements in twin sisters with CDG syndrome type 1. Despite progressive deterioration of electroretinographic findings, good central vision was preserved over 14 years.

The authors thank G Van Parrijs for the preparation of the figures.

1 Jaeken J, Carchon H, Stibler H. The carbohydrate-deficient glycoprotein syndromes. Pre-Golgi and Golgi disorders? Glycobiology 1993;3:423-8.

2 Jaeken J, Stibler H, Hagberg B. The carbohydrate-deficient glycoprotein syndrome. A new inherited multisystemic disease with severe nervous sytem involvement. Acta Paediatr Scand Suppl 375: (monograph) 1991.

3 Kristiansson B, Andersson M, Tonnby B, Hagberg B. Disialotransferrin developmental deficiency syndrome. Arch Dis Child 1989;64:71-6.

4 Stromland K, Hagberg B, Kristiansson B. Ocular pathology in disialotransferrin developmental deficiency syndrome. in disialotransferrin developmental defici

5 Andreasson S, Blennow G, Ehinger B, Stromland K. Full field electroretinograms in patients with the carbohydratedeficient glycoprotein syndrome. Am $\mathcal{F}$ Ophthalmol 1991; 112:83-6.

6 Fiumara A, Barone R, Buttitta P, Di Piedro M, Scuderi A, Nigro F, et al. Carbohydrate deficient glycoprotein syndrome type 1: ophthalmic aspects in four Sicilian patients. Br ₹ Ophthalmol 1994;78:845-6.

7 Jaeken J, Vanderschueren-Lodeweyckx M, Casaer P, Snoeck L, Corbeel L, Eggermont E, et al. Familial psychomotor retardation with markedly fluctuating serum prolactin, FSH and GH levels, partial TBG deficiency, increased serum arylsulfatase $A$ and increased CSF protein: a new syndrome? Pediatr Res 1980;14:179.

8 Jaeken J, van Eijk HG, van der Heul C, Corbeel L, Eeckels R., Eggermont E. Sialic acid-deficient serum and cerebrospinal fluid transferrin in a newly recognized genetic syndrome. Clin Chim Acta 1984;144:245-7.

9 Jaeken J, Eggermont E, Stibler H. An apparent homozygous $\mathrm{X}$-linked disorder with carbohydrate deficient serum glycoproteins. Lancet 1987; iii: 1398.

10 Alfieri R, Sole P. Adapto-électrorétinogramme en lumière monochromatique chez l'homme et analyse formelle des potentiels rétiniens. CR Soc Biol (Paris) 1966;160:1882-6.

11 Standard for Clinical Electroretinography-International Standardization Committee. Arch Ophthalmol 1989;107: 816-9. 